\title{
Flow of power-law fluids in self-affine fracture channels
}

\author{
Yiguang Yan $^{1,2}$ and Joel Koplik ${ }^{1,3}$ \\ Benjamin Levich Institute ${ }^{1}$ and Departments of Mechanical Engineering ${ }^{2}$ and Physics ${ }^{3}$, \\ City College of the City University of New York, New York, NY, 10031
}

(Dated: April 21, 2022)

\begin{abstract}
The two-dimensional pressure driven flow of non-Newtonian power-law fluids in self-affine fracture channels at finite Reynolds number is calculated. The channels have constant mean aperture and two values $\zeta=0.5$ and 0.8 of the Hurst exponent are considered. The calculation is based on the lattice-Boltzmann method, using a novel method to obtain a power-law variation in viscosity, and the behavior of shear-thinning, Newtonian and shear-thickening liquids is compared. Local aspects of the flow fields, such as maximum velocity and pressure fluctuations, were studied, and the nonNewtonian fluids were compared to the (previously-studied) newtonian case. The permeability results may be collapsed into a master curve of friction factor vs. Reynolds number using a scaling similar to that employed for porous media flow, and exhibits a transition from a linear regime to a more rapid variation at $\mathrm{Re}$ increases.
\end{abstract}

\section{INTRODUCTION}

An understanding of flow and transport processes in geologically disordered media is necessary for the efficient extraction of fluids from underground hydrocarbon reservoirs. Situations where flow proceeds through networks of connected fractures are particularly attractive, because the throughput is generally much higher than may be achieved through intergranular porosity alone [1, 2, 3, 4]. An important feature of subsurface fractures, which considerably complicates the problem, is that the surfaces of naturally fractures rocks are not smooth or even randomly rough, but rather are highly correlated self-affine fractals [5]. A second complication in the analysis is that typical reservoir fluids are often complicated mixtures, which exhibit non-Newtonian flow behaviors such as shear-thinning or shear-thickening. Yet a third difficulty is that the subsurface fracture flow often involves much higher velocities than in the intergranular case, and the common simplification of low-Reynolds number linear flow is inapplicable.

In this paper we use lattice Boltzmann calculations to elucidate the combined effects of self-affinity, non-linear rheology and finite inertia in fluid flow through a single fracture. Previous authors have considered subsets of these complications, but not all three simultaneously. The flow of Newtonian fluids in self-affine fractures at both low [6, 7] and finite [8] Re has an extensive literature. Some controlled experiments on shear-thinning fluids in self-affine fractures at low $R e$ have been reported [9]. Lastly, experiments and phenomenological models for non-linear fluid motion in intergranular porous media at various $R e$ are available [10]. We anticipate that flow in a fracture can be characterized in a manner similar to the latter problem, since in both cases the key effect is that the random solid boundary of the flow domain causes streamlines to wind around. One simplification which we can exploit, however, is to focus on twodimensional flows. It is well known that the flow of a single fluid in a straight channel differs only in detail between two and three-dimensional cases, and furthermore, in porous media flow in the analogous intergranular case, one sees the same flow laws for both two and three dimensional geometries.

The approach taken in the paper follows the lines of our previous studies of permeability [6] and transport [7] in self-affine fractures based on the lattice-Boltzmann method, along with a procedure for incorporating powerlaw viscosity variation similar to that developed previously [11]. The discussion of inertial effects is influenced by previous studies for the case of a Newtonian fluid in intergranular porous media [8]. The fracture surface is generated numerically by a Fourier transform algorithm and discretized on the regular lattice used in the flow problem. The upper and lower fracture surfaces bound the allowed nodes in the flow domain, a bounce-back condition enforces the no-slip boundary condition, and constant forcing provides a pressure driven flow. For powerlaw fluids, the lattice-Boltzmann relaxation time is adjusted locally in space and time to provide the desired relation between stress and strain. The relation between imposed pressure drop and total fluid flux provides the permeability, and the local flow fields are analyzed to discuss the velocity, pressure and shear stress variations. Some background on the flow geometry and calculational method is presented in Section $\amalg$ the local analysis of the flow fields in Sec. III, the discussion of permeability is in Sec. [V] and we summarize in Sec. V]

\section{BACKGROUND}

\section{A. Self-affine roughness}

In this subsection we review the characterization of self-affine fractures and their numerical implementation. We consider a fracture surface without overhangs, i.e., the surface height $h(x, y)$ is a single-valued function of the two coordinates $\mathbf{r}=(x, y)$ lying in the mean plane of the surface. A self-affine fractal surface is one which displays different scaling along the different spatial directions [12], 
a statistical self-similarity under the transformation

$$
x \rightarrow \lambda x \text { and } y \rightarrow \lambda y \Rightarrow h(\mathbf{r}) \rightarrow \lambda^{\zeta} h(\mathbf{r})
$$

where $\zeta$ is the Hurst or roughness exponent. Observations of a variety of naturally fractured rock surfaces in different fracture modes yield just two common values of $\zeta$, approximately 0.5 and 0.8 . We further assume that the surface has spatial isotropy in its mean plane. The surface is further characterized by the amplitude of the roughness, or equivalently the prefactor $C_{0}$ in the heightheight correlation function,

$$
\left\langle[h(\mathbf{r}+\boldsymbol{\Delta})-h(\mathbf{r})]^{2}\right\rangle=C_{0}(|\boldsymbol{\Delta}| / \ell)^{2 \zeta}
$$

where the intrinsic length scale $\ell$ might be the grain size in experiment or the lattice spacing in a calculation. In practice we generate self-affine surfaces using the a Fourier synthesis method [13] as in [6].

A self-affine fracture channel is made of two complementary self-affine surfaces separated by a gap, and in some cases the surfaces are shifted relative to each other parallel to the mean plane. The statistical properties of the fracture are specified by the Hurst exponent, the mean aperture between two surfaces, the shift distance, if any, and by the amplitude of the roughness. The height fluctuations of a single self-affine surface increase with its lateral extent $L$, so that the difference between the maximum and minimum heights scales as $(L / \ell)^{\zeta}$, and we consider the limit $H \ll R<L$, as shown for a typical fracture in Fig. [1.

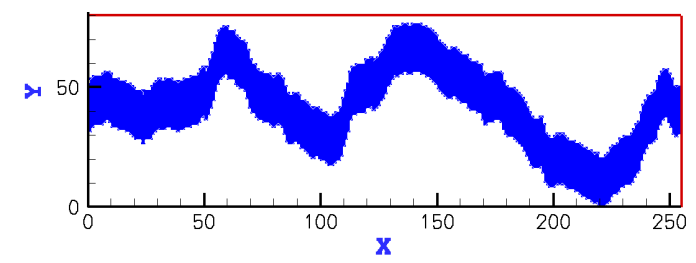

FIG. 1: Geometry of a typical self-affine fracture composed of two complimentary self-affine surfaces with $\zeta=0.8$.

Note that the effective flow diameter of the fracture varies along its length and can be much smaller than the mean aperture, due to the tortuosity of the channel. When a lateral shift is present, the aperture varies locally as well, and furthermore if $H$ is too small the sides of the fracture may overlap.

\section{B. The lattice-Boltzmann method}

Since the flow domain is bounded by highly irregular surfaces, the lattice Boltzmann method [14] is particularly convenient for fluid mechanical calculations, since the excluded solid region may be simply specified by a mask. If $f_{i}(\mathbf{x}, t)$ is the velocity distribution function (VDF) for particles moving in direction $i$ at lattice site $\mathbf{x}$ at time $t$, then the discrete Boltzmann equation which evolves the distribution is

$$
f_{i}\left(\mathbf{x}+\mathbf{e}_{i}, t+1\right)=f_{i}(\mathbf{x}, t)+\Omega_{i}(f(\mathbf{x}, t)),
$$

Here the $\mathbf{e}_{i}$ are unit lattice vectors, the lattice spacing and the time step are both set equal to one, $\Omega_{i}(f(\mathbf{x}, t))$ is collision operator which redistributes the VDF along different directions, and the spatial and temporal step discretizes in single unit. To recover the Navier-Stokes equation of fluid flow starting from Boltzmann equation, moments of the VDF satisfy the constraints

$\rho=\Sigma_{i} f_{i} \quad \rho \mathbf{u}=\sum_{i} f_{i} \mathbf{e}_{i} \quad \boldsymbol{\sigma}=-\rho c_{s}^{2} \mathbf{I}-\left(1-\frac{1}{2 \tau}\right) \sum_{i} f_{i} \mathbf{e}_{i} \mathbf{e}_{i}$

which relate the distribution function to the continuum density, velocity and stress fields, and where $c_{s}$ is the sound speed. The collision operator is treated in the BGK approximation using a single characteristic relaxation time $\tau$,

$$
\Omega_{i}(f(\mathbf{x}, t))=-\frac{1}{\tau}\left(f_{i}(\mathbf{x}, t)-f_{i}^{e q}(\mathbf{x}, t)\right),
$$

where $f_{i}^{e q}(\mathbf{x}, t)$ is equilibrium distribution function. The relaxation time $\tau$ is related to the kinematic viscosity of the fluid by $\nu=(2 \tau-1) / 6$. To simulate a constant pressure gradient we add a a constant body force term to the right hand side of eq[3 equation eq 3 and 5 to NavierStokes equation. More details may be found in [14], and a recent review of flow simulations in this context is presented by Verberg and Ladd[15].

\section{Power-law fluids}

The basic idea in extending the lattice Boltzmann method to power-law fluids was presented by Aharonov and Rothman [16], and consists of adjusting the relaxation time $\tau$ locally so as to achieve the desired ratio of stress to strain rate. Here we consider power-law fluids using a generalized Newtonian model, as in [11], where the relation between the stress tensor $\sigma_{\alpha \beta}$ and the strain rate tensor $D_{\alpha \beta}=1 / 2\left(\left(\partial_{\beta} u_{\alpha}+\partial_{\alpha} u_{\beta}\right)\right.$ is similar to that for Newtonian fluids, $\sigma_{\alpha \beta}=2 \mu D_{\alpha \beta}$, but the local viscosity $\mu$ is a function of the invariants of the strain rate tensor. We consider power-law fluids, $\mu=m \dot{\gamma}^{n-1}$, where the case $0<n<1$ corresponds to shear-thinning, $n>1$ corresponds to shear-thickening, and $n=1$ recovers linear Newtonian fluids, where the local shear rate $\dot{\gamma}$ is related to the second invariant of $D_{i j}$ via $\dot{\gamma}=(2 \mathbf{D}: \mathbf{D})^{1 / 2}$. The procedure in [11] was to obtain the strain rate tensor by numerical differentiation of the previously calculated velocity field, then determine the appropriate local viscosity and thence the local relaxation time. Here we adopt a different procedure: in the lattice Boltzmann method the strain rate tensor is directly related to the velocity 
distribution function by $[17$

$$
D_{\alpha \beta}=-\frac{3}{2 \rho \tau} \sum_{i}\left(f_{i}-f_{i}^{e q}\right) \mathbf{e}_{i \alpha} \mathbf{e}_{i \beta}
$$

which should in turn equal $\sigma_{\alpha \beta} / 2 \mu$, there is a constraint on the $f_{i}$ which is solved by iteration.

To validate the formulation of power-law fluids given above, we calculate the velocity profile for pressuredriven flow in a smooth-walled channel of constant aperture (a Hele-Shaw cell), which may be compared to an analytic solution of the Navier-Stokes equation. Applying a pressure gradient $\Delta P / L=-G$ in the $x$-direction, the velocity for a power-law fluid with rheological parameters $m, n>0$ as above in a channel of width $H$ is

$u_{x}(y)=\frac{n}{n+1}\left(\frac{G}{m}\right)^{1 / n}\left(\left|\frac{H}{2}\right|^{(n+1) / n}-\left|\frac{H}{2}-y\right|^{(n+1) / n}\right)$,

We also record the mean velocity $\bar{u}$ and the fluid flux $Q$ (per unit length in the passive third direction), which will be useful below:

$$
Q=H \bar{u}=\int_{0}^{H} d y u_{x}(y)=\frac{n}{2 n+1}\left(\frac{H^{2}}{2}\right)\left(\frac{G H}{2 m}\right)^{1 / n}
$$

In the simulation, we begin with zero velocity and integrate Eq. (3) to steady state, using the convergence criterion

$$
\epsilon=\sum_{x} \frac{\|u(\mathbf{x}, t)-u(\mathbf{x}, t-1)\|}{\|u(\mathbf{x}, t)\|}<1.0 \times 10^{-6}
$$

For power-law indices $n=0.75,1.0$ and $1.25, m=0.01$, and pressure gradient $G=1 \times 10^{-6}$ we obtain the profiles shown in Fig. 2, which agree with theory. In practice, as with any numerical method, computational instabilities may occur for substantially different values of the pressure gradient and fluid index, but the algorithm could be extended there using techniques such as multi-time step relaxation for the local shear viscosity [18].

\section{LOCAL ANALYSIS OF THE FLOW FIELD}

We wish to examine how the local flow behavior varies with the rheology of the fluid, at different geometrical features of a self-affine channel. We focus on a single realization of the fracture, shown in Fig. 1, and vary the power law index $n$ and the pressure gradient $G$. The complete simulation box has length $L=256$ in the flow direction and width $W=80$, in terms of the (unit) lattice spacing, and the (constant) vertical aperture is $H=20$. A uniform pressure gradient is applied everywhere along the channel, as above, and periodic boundadry conditions are applied in the flow direction. Local minimum in the effective width (normal to the average flow) occur around $x=55,110$, and 240 where mass conservation implies the
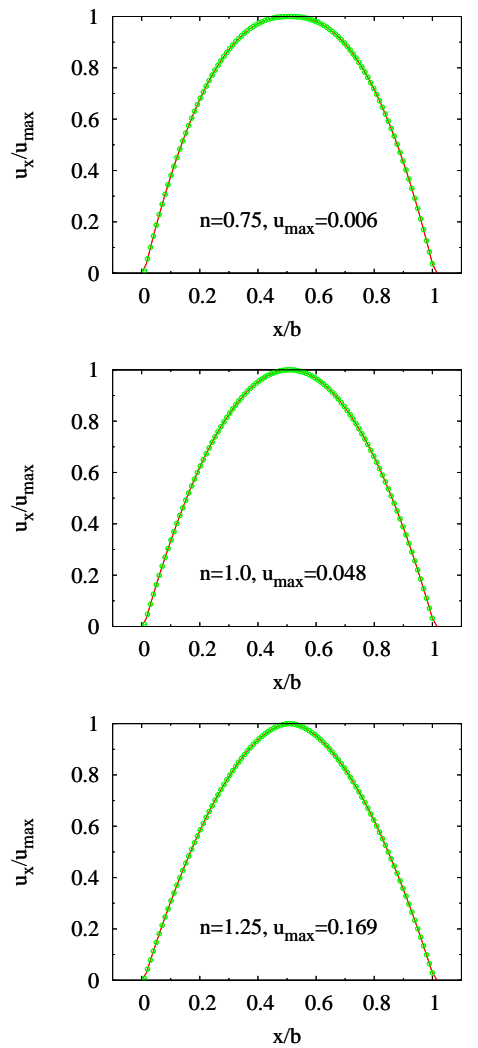

FIG. 2: Velocity profiles of power-law fluids with $m=$ $0.01, n=0.75,1.0,1.25$ in a Hele-Shaw cell with pressure gradient $G=1 \times 10^{-6}$. The points are simulation results while the solid lines are the analytical solution in (17). The maximum velocities for the three fluids are $u_{\max }=$ $0.006,0.048,0.169$, respectively.

velocity magnitude will be a maximum, irrespective of the rheology of the fluid. In Fig. 3, we show velocity fields and streamlines for the three fluids along a segment of the fracture channel $20 \lesssim x \lesssim 100$ in Fig. 1 which includes a constriction, for applied pressure gradient $G=1 \times 10^{-6}$. As we see, the streamlines are tortuous and very roughly follow the channel walls, although recirculating eddies (closed vortices) may occur where the channel exhibits side branches or dead-end regions. Indeed, at the present flow rate an eddy appears in the shear-thickening case but not the others, presumably because the velocites are higher in that case.

\section{A. Velocity field}

First we examine the variation of maximum absolute velocity along the channel, in order to show how the fluid rheology influences the earlier results of Skjetne et al. [8] for the Newtonian case. More precisely, for each $x$ along the channel we compute the maximum over $y$ of $|\mathbf{u}(x, y)|$, although we would have reached the same qual- 

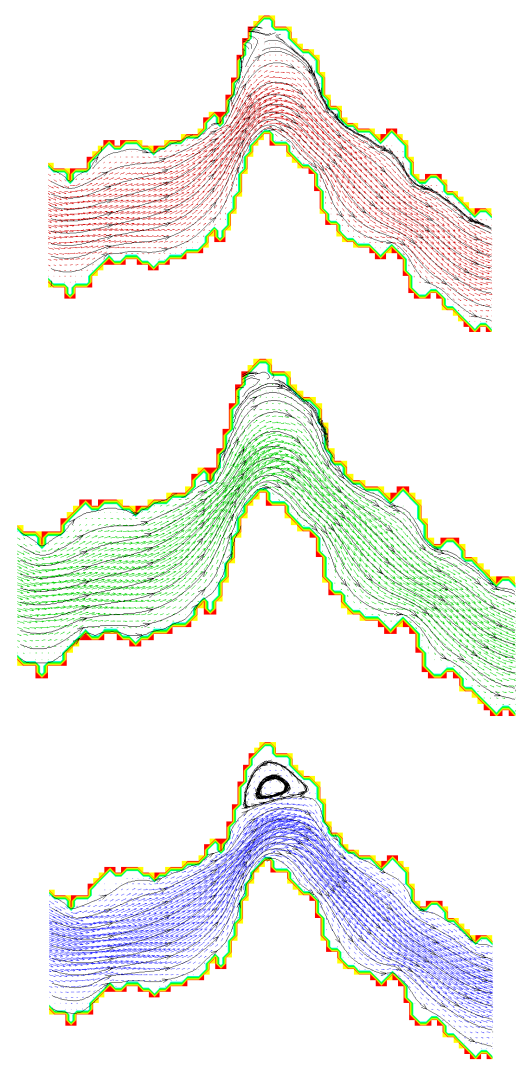

FIG. 3: Segment of Velocity vector field with streamlines of the flow for power-law fluid with $m=0.01, n=$ 0.75 (top), 1.0 (middle), 1.25 (bottom) and the pressure gradient applied is $G=1 \times 10^{-6}$. The segment extends from $x=20$ to $x=100$.

itative conclusions had we considered the maximum over $y$ of $u_{x}(x, y)$. Calculations were performed for three values of the pressure gradient, $G=1 \times 10^{-6}, 5 \times 10^{-5}$ and $2 \times 10^{-4}$, which correspond to Reynolds numbers $R e=0.95,37.0$ and 92.7, respectively, for the Newtonian fluid. Since the viscosity varies within the channel for the shear thinning and thickening fluids, there is no unique definition of $R e$ in those cases, although a convenient choice will be introduced in Section IV for scaling purposes.

The resulting plots of maximum velocity are shown in Fig. 4, where each velocity is normalized by the average streamwise flow velocity $\overline{u_{x}}$ (referred to as the interstitial velocity $u^{*}$ in [8]), which equals the flux divided by the channel width. Obvious peaks appear at the positions of the visible constrictions in the channel near $x=55,110$ and 240, reflecting the narrowed aperture there. The normalized peak heights are fairly insensitive to the Reynolds number, although away from the peaks the trend is for maximum velocity to increases with $R e$. Note that for a flat channel, the the normalized maximum absolute velocity would equal 1.5 , so the values of 5 or more seen here are a substantial enhancement. The peaks are not all closely correlated with channel constrictions, however: near $x=70$ and 130 maximum velocity peaks occur, but at these locations the channel is expanding just downstram of a constriction. It is also possible to calculate a "maximum velocity trajectory", following [8], as the set of $(x, y)$ gridpoints which at each $x$ has the $y$-value corresponding to the position where the maximum velocity occurs. For the most part our observations concerning the behavior of these trajectories is similar to that reported in this reference, but we do not observe the line-length of this trajectory decreasing monotonically with $R e$.
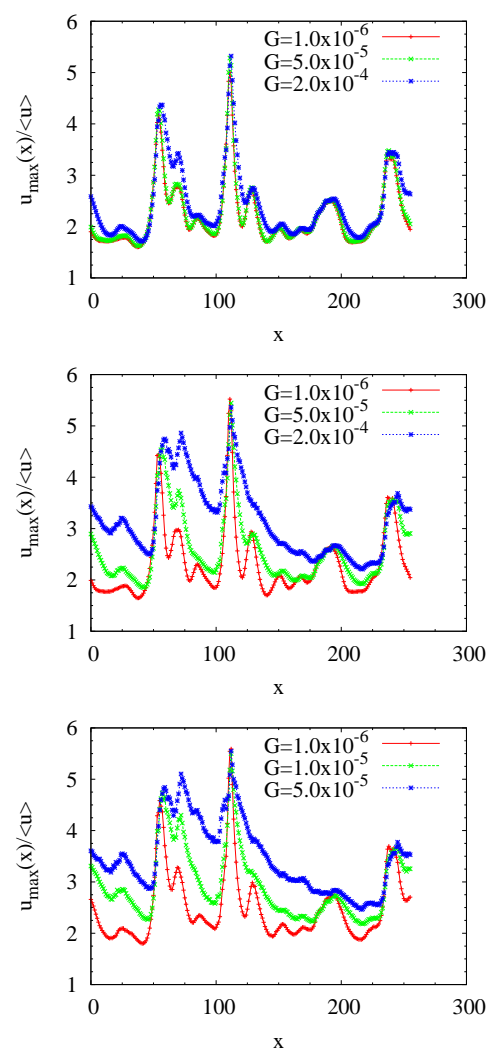

FIG. 4: Maximum absolute velocity along the fracture channel for shear- thinning(top, $n=0.75)$,Newtonian(middle, $n=$ 1.0), and shear- thickening( bottom, $n=1.25$ ) fluids for various applied pressure gradient $G$. Each maximum velocity curve is normalized by the corresponding $\overline{u_{x}}$, the average flow velocity in the $x$-direction.

Comparing the other fluids to the Newtonian case, we see in Fig. 4 that the global maximum absolute velocity always occurs at the narrowest constriction near $x=110$ and the other primary peaks always occur at the same positions, $x=55$ and 240, as well. Furthermore, each peak has roughly the same (normalized) velocity value. In the shear-thinning case, both the variation in $x$ away from the peaks/constrictions and the variation with pressure gradient are weaker than in the other cases, which may be attributed to the fact that typical velocities in the fracture are smaller in this case, and inertial effects 
play a weaker role. In the shear-thickening case, where typical velocites are larger, the maximum velocity values are larger off the peaks values, and furthermore exhibits rather more variation with $x$ and $R e$ than the other fluids.

The probablity distribution of velocity magnitudes is also of interest [8], since the presence of low and high velocity components strongly influences mixing processes and transport of passive tracers and suspended particles [19]. Histograms of the observed absolute value of the velocity for the three fluids at various pressure gradients

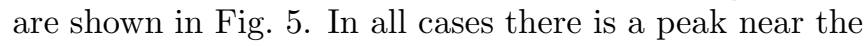
origin, which reflects the numerous low-velocity zones in the crevasses at the fractures walls, along with a highervelocity peak resulting from the rapid flow in the channel constrictions. The latter moves out to higher values as the pressure gradient increases (note the normalization by $\overline{u_{x}}$ in the figure) Once again, the shear-thickening case behaves somewhat differently than the other two fluids, showing a less prominent and broader "constriction peak," and more variation with $G$.
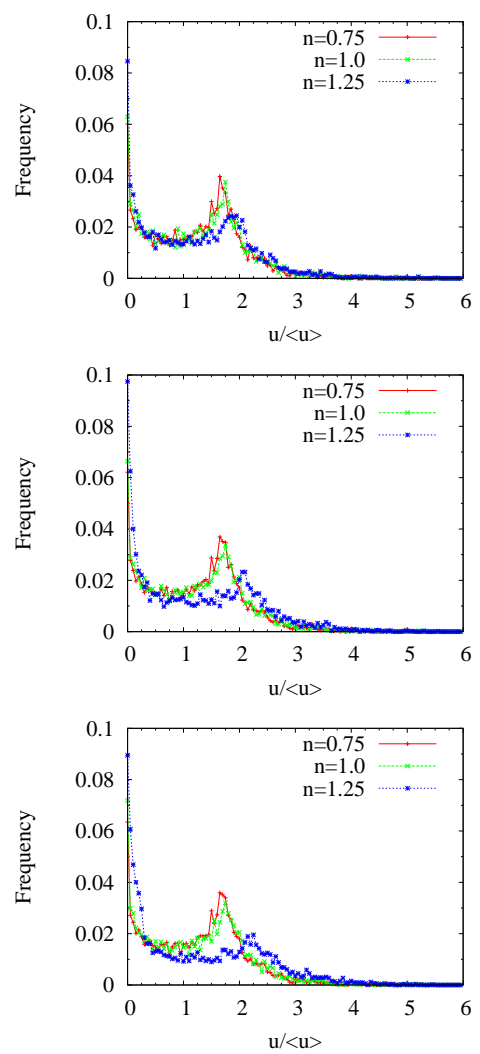

FIG. 5: Distribution of normalized absolute velocity in the whole self-affine fracture flow domain for different power-law fluids with pressure gradients $G=1.0 e-6,5.0 e-6$ and $1.0 e-5$ (top to bottom).

\section{B. Pressure and stress field}

The distribution of pressure and stress in the fluid are important for non-Newtonian rheology, and in considering possible erosive processes on the fracture walls. To contrast the behavior of the different fluids, Fig. 6 shows the pressure "fluctuations" along the channel for the three power-law fluids $n=0.75,1.0$ and 1.25 . The fluctuation $p^{\prime}$ is the deviation in pressure from the imposed linear gradient, which would vanish identically in a Hele Shaw geometry. In the figure, the fluctuation has been normalized by the imposed pressure difference, $\Delta p=G L$, and averaged over the channel width. For all three fluids, the pressure fluctuation are most significant in the vicinity of the main constrictions in the channel where the fluid accelerates, rising just before each constriction's location and dropping rapidly as it is traversed. Some additional structure arises at positions $x$ of bends in the flow path, another source of fluid acceleration. Again, the shear-thinning and Newtonian fluids behave somewhat similarly, while the variation is strongest in the shear-thickening case.

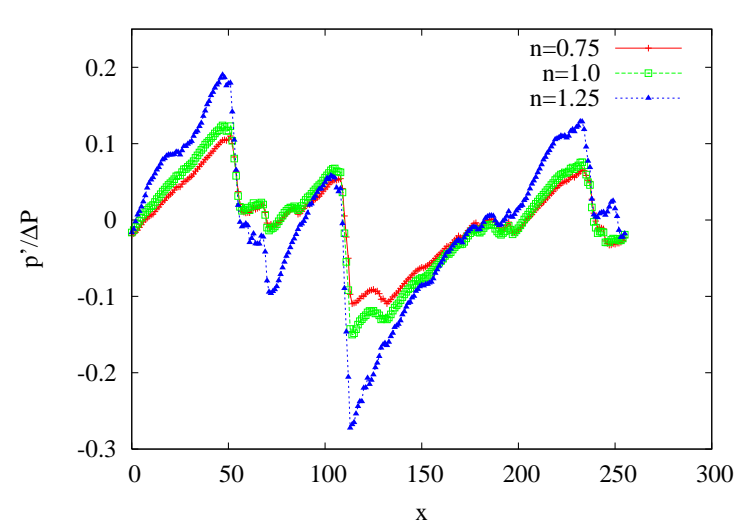

FIG. 6: Normalized pressure fluctuation along the channel for different power-law fluids, at pressure gradient $G=1.0 e-5$.

The variation in fluctuation with imposed gradient is shown in Fig. 7, and indicates the expected general increase in magnitude with $G$ along the channel.

To assess the effects of the flow on the fracture wall, we first calculate the average force exerted by the fluid on the wall,

$$
\mathbf{F}=\frac{1}{L} \int d \ell \hat{\boldsymbol{n}} \cdot \boldsymbol{\sigma}
$$

where the integral runs over the fracture surface (a curve in this two-dimensional calculation), and $\hat{\mathbf{n}}$ is the local normal to the wall. The force is then decomposed into $x$ and $y$ components, representing the average drag and thrust on the wall, respectively, and then normalized by a typical inertial pressure $\rho \bar{u}^{2} / 2$ times the nominal surface 

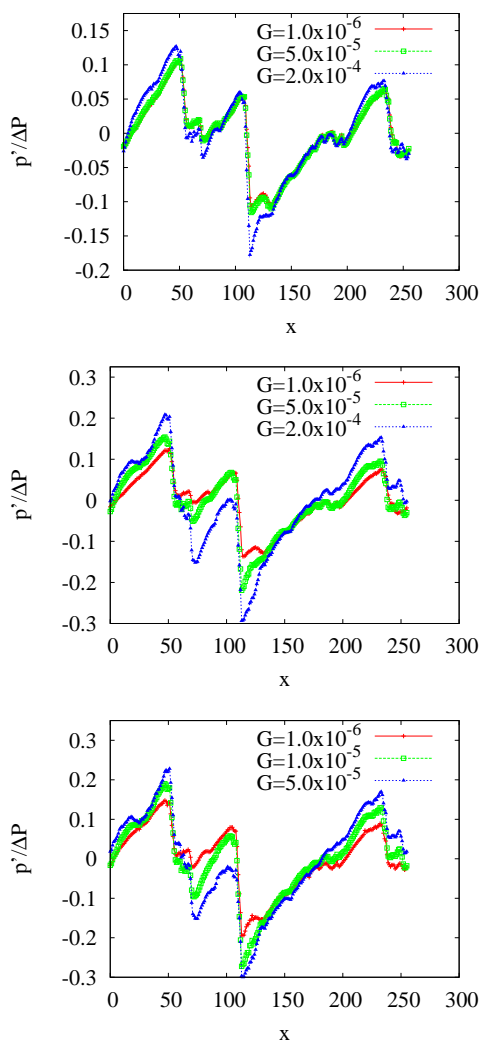

FIG. 7: Pressure fluctuations of different fluids with power $n=0.75,1.0,1.25$ and $m=0.01$ along a self-affine fracture channel under different applied pressure gradients.

area $L \times 1$, to give drag and thrust coefficients

$$
d=\frac{F_{x}}{L \rho \bar{u}^{2} / 2}, \quad t=\frac{F_{y}}{L \rho \bar{u}^{2} / 2} .
$$

Note that aside from the (reasonable) use of the inertial pressure, the remainder of the normalization is somewhat arbitrary but a fixed constant for each fracture, and mainly serves to provide dimensionless drag and thrust coefficients. The drag and thrust forces for the lower and upper walls of the channel are similar but not identical because of the asymmetry of the fluid-solid boundary, and for definiteness we present only the forces on the lower wall.

The results of calculating the drag and thrust coefficients is shown in Figs. 8 and 9 for the three fluids with exponents $n=0.75,1.0,1.25$. In all cases, the coefficients exhibit simple power-law behavior, provided $G$ is not too large, and the transition to a different behavior at larger $G$ may be associated with the onset of inertial effects (see the following section). This form of scaling behavior result is consistent with the experimental results reported in [10], and the values of the slopes found in the log-log plots in the low- $G$ range, $-1.67,-1.02,-0.62$ for $n=0.75$, 1.0 and 1.25 , respectively, for both drag and thrust, may be understood from the following argument.

If inertial effects are absent, one expects the scaling behavior in a rough channel to be the same as in a straight channel. In that case, from Eq. (7) one has $u \sim G^{1 / n}$, and therefore $\nabla u \sim G^{1 / n}$ as well, so that $\mu \sim|\nabla u|^{n-1} \sim$ $G^{(n-1) / n}$. The drag and thrust forces are proportional to the stress, $\sigma \sim \mu \nabla u \sim G^{(n-1) / n+1 / n} \sim G^{1}$. The drag and thrust coefficients are then $d, t \sim F_{x, y} / \bar{u}^{2} \sim \sigma / u^{2} \sim$ $G^{1-2 / n}$, giving exponents $-5 / 3,-1$ and $-3 / 5$, respectively, for the three fluids.

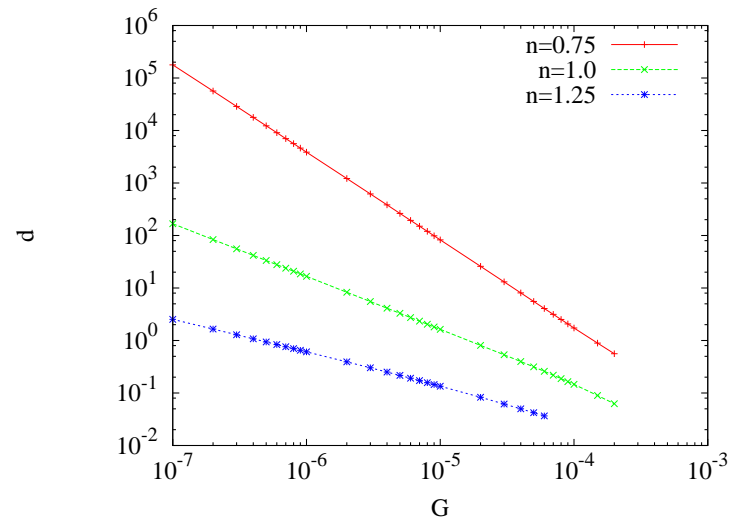

FIG. 8: Drag factor $d$ for power-law fluids in a self-affine fracture channel as a function of applied pressure gradient.

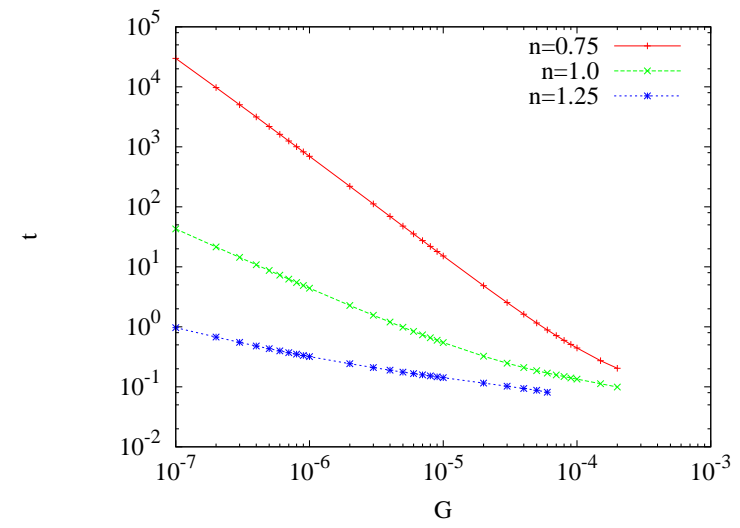

FIG. 9: Thrust factor $t$ for power-law fluids in a self-affine fracture channel as a function of applied pressure gradient.

\section{PERMEABILITY}

Next we consider global behavior - the permeability of a self-affine fracture channel. Our discussion is colored by analogies to flow in intergranular porous media, so we first recall the situation in that system [20]. For Newtonian fluids in the low Reynolds number limit, the definition of intergranular permeability is given by Darcy's 
law, $\langle\mathbf{u}\rangle=-(k / \mu) \nabla p$, where $\langle\mathbf{u}\rangle$ is the average flow velocity and $p$ the average pressure. The average in question could be a volume average or an ensemble average, and for a flow which is macrosopically unidirectional, an operational definition of permeability is $k=\mu Q L / A \Delta p$ where $Q$ is the flux through a sample of cross-sectional area $A$ and length $L$. In a two-dimensional situation, the area is replaced by the width $W$, and $Q$ is the flow per unit length in the third direction. A definition identical to the latter case may be used for the permeability of low Reynolds number Newtonian flow in a fracture. Both finite Reynolds number flow and non-Newtonian fluid rheology modify this description. We first consider the effects of inertia, and then examine how permability relates to the fracture morphology.

\section{A. Inertial effects}

At higher flow rates when inertial effects appear, the relation between pressure difference and average velocity or flux becomes nonlinear and one may write

$$
\Delta p=\alpha Q+\left[\beta Q^{2} \text { or } \gamma Q^{3}\right]
$$

where $\alpha$ incorporates the Darcy permeability, and the term in brackets is the inertial correction, with $\alpha, \beta>0$. At high $Q$ the quadratic or "Forchheimer" term applies, but in the transitional region where the Reynolds number is small but finite, a cubic dependence is found. This picture is supported by experiments, analytic calculations, and numerical simulations [21].

The flow of a Newtonian fluid in a self-affine fracture can be described in identical terms, as shown by the numerical simulations of Skjetne et al. [8] which exhibit the same transitions between flow regimes indicated in Eq. (12). In extending the discussion to power-law fluids, the first issue is to choose the appropriate power of $Q$. The exact solutions for Hele Shaw flow given in Eq. (8) have the scaling behavior $G \sim Q^{n}$ where $G$ is the applied pressure gradient (the relevant pressure for macroscopic behavior) and $n$ the power-law index. In a rough fracture, one would naturally expect an identical relation, albeit with a modified coefficient, at low $G$, and then at larger $G$ inertial effects would be expected to produce (positive) terms involving higher powers of $Q$. To test this idea, note that we are concerned here with the statistical behavior of self-affine fractures, rather than the details of flow in one particular geometry which was relevant in the previous section, so an ensemble average over six realizations of the fracture surface is used. The simulation results are shown in Fig. 10 and indeed show a $G \sim Q^{n}$ scaling behavior at low $G$. The Newtonian $n=1$ plot shows this behavior clearly since $G / Q^{n}$ is constant at low $Q$, whereas in the other cases, the expected behavior is present at sufficently small $Q$ as indicated in the alternative plots in the insets of $G$ vs. $Q$. The need for different plotting variables arises becuase in the
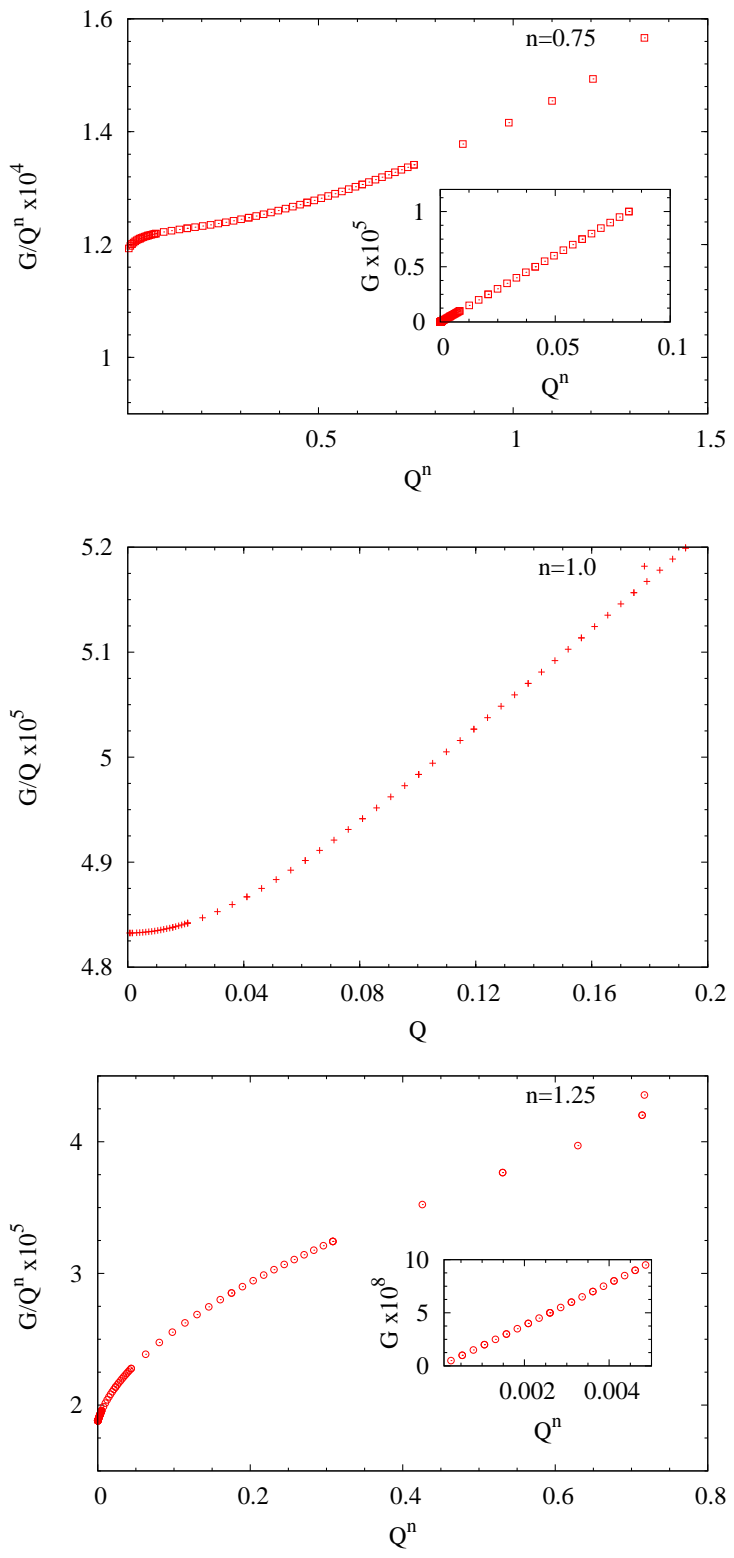

FIG. 10: Relation between imposed pressure gradient and fluid flux for power law fluids: $n=0.75,1.0$ and 1.25 (top to bottom).

non-Newtonian cases, the flow rate fluctuates substantially at low $G$ and division by $Q^{n}$ is numerically unstable. Beyond the quasi-linear regime, the Newtonian case shows the expected trasition to a Forchheimer flow regime $G \sim Q^{2}$ at larger forcing, and the shear-tickening fluid shows a somewhat analogous behavior $G \sim Q^{2 n}$. The shear-thinning fluid is not described by a simple power law at large $G$, and we are not aware of any theoretical treatment of this problem, so we simply report the numerical results.

To understand the numerical coefficient in the flow results, the fracture-modified Darcy permeability, we again 


\begin{tabular}{|l|c|c|}
\hline fluid index & $k_{0}$ & $k$ \\
\hline$n=0.75$ & 2.99 & 0.373 \\
\hline$n=1.0$ & 33.2 & 4.73 \\
\hline$n=1.25$ & 142 & 20.6 \\
\hline
\end{tabular}

TABLE I: Effect of roughness and tortuosity on the low Reynolds number permeabilty: $k_{0}$ and $k$ are the permeabilitites (defined in Eq. (13) for a Hele Shaw cell and a self-affine fracture of the same mean aperture, respectively.

refer to the Hele Shaw case and define

$$
k=\frac{m^{1 / n} \bar{u}}{G^{1 / n}} .
$$

Since the roughness and tortuosity of the fracture cause the stramlines to bend and viscous dissipation to increase, the permeability should be reduced compared to a smooth and flat Hele Shaw geometry of the same aperture. In Table I, the various permeabilites are compared, and a reduction by a factor $6-7$ is found.

So far, we have expressed the pressure gradient $G$ in terms of the flux $Q$, because these quantities are well defined in the present simulations. However, for general purposes It is preferable to use a dimensionless quantity such as the Reynolds number as the independent variable, but the definition of $R e$ for power-law fluids is not entirely obvious for power-law fluids because the the viscosity varies over the flow domain. One way to combine the results for different fluids is based on an analogy to the friction factor scaling laws for flow in pipes originally due to Nikaradze 22], which can be extended to non-Newtonian fluids as shown by Metzner [23]. Recall that for unidirectional flow of a Newtonian fluid of viscosity $\mu$ in a pipe of diameter $D$, the mean velocity is $\bar{u}=G D^{2} / 32 \mu$ and the shear stress at the wall is $\tau_{w}=G D / 4$, so if one defines the conventional friction factor as $f=\tau_{w} / \frac{1}{2} \rho \bar{u}^{2}$, then one finds $f=16 / R e$ where $R e=\rho \bar{u} D / \mu$. Experiments follow this scaling law up to a value of $R e$ that depends on the roughness of the pipe, and at larger values of Re, $f$ levels off. An analogous calculation for Hele Shaw flow using the aperture $H$ instead of the diameter $D$ gives $\tau_{w}=G H / 2$ and $f=12 / R e$. The power-law generalization is to use the latter form for $\tau_{w}$, along with Eq. (8) to express the pressure gradient in terms of the mean velocity, and yields

$$
f=\frac{12}{R e} \quad \text { if } \quad R e \equiv 6 \rho \bar{u}^{2-n} H^{n} / m^{\prime},
$$

where $m^{\prime}=m(2(2 n+1) / n)^{n}$. This choice of variables is not the last word, because in the analogous interganular porous medium case where a similar approach has been taken [10], extra constant factors such as functions of the porosity or the "dynamic specific surface area" are introduced into the friction factor and Reynolds number definitions to promote data collapse. It is not clear how such ad hoc factors might be interjected here, so instead we collapse the data using a simple constant factor which varies from fluid to fluid, and the result is shown in Fig. 11. Two different values of the Hurst exponent are shown, and in both cases we see an $F \sim 1 /$ Re scaling at low $R e$, a transition at $R e \sim 1-10$ and perhaps a constant friction factor at larger Re. Unfortunately, the calculations cannot be extended into the latter regime using the present method (a particluar implementation of the lattice Bolzmann technique) becuase numerical instabilites arise.

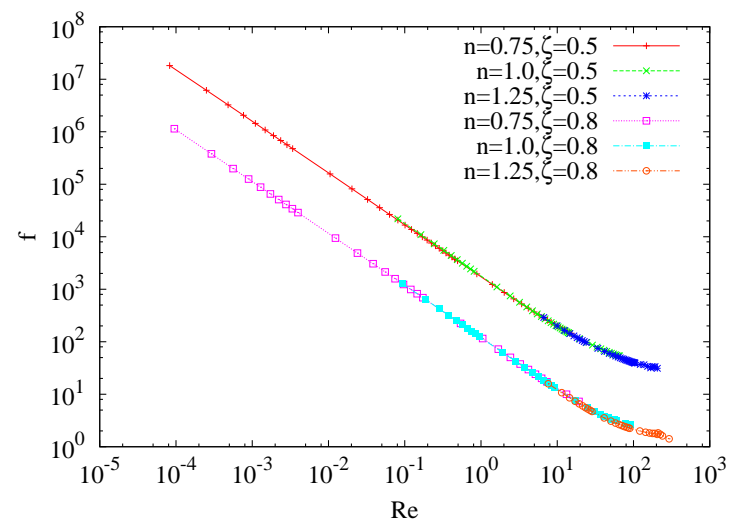

FIG. 11: Friction factor of self-affine fracture channels of Hurst exponent $\zeta=0.5$ and 0.8 as a function of Reynolds number defined as in 14 for power-law fluids with $n=$ $0.75,1.0,1.25$.

\section{B. Morphology effects}

We now consider how the geometry of the fracture effects the (low-Reynolds number) permeability for the various fluids considered. First we investigate the effect of the Hurst exponent $\zeta$ on the permeability, and to simplify the analysis we consider a fracture channel with one selfaffine wall and one flat wall, as in [6]. For a fixed presure gradient $G$, we compute the flux as a function of the channel length $L$ for the three fluids, and in Fig. 12, we first show the flow rate depletion $\left(Q_{0}-Q\right) / Q_{0}$ vs. $L$ for a fracture with $\zeta=0.8$. Here $Q_{0}$ is the flux through a flatwalled channel fo the same mean aperture. Increasing the length allows for more fluctuation in the channel width (see Eq. (2) which increases the tortuosity and tends to decrease the flux. If the Hurst exponent of the channel's rough wall is instead $\zeta=0.5$, the three fluids again behave quite similarly, so it suffices to compare the behavior of different Hurst exponents for a single case, and in the lower panel of the figure we plot the flux depletions for the two exponents for the shear-thinning case. The fact that the flux depletion is greater for the $\zeta=0.8$ channel may be explained by noting that this exponent value corresponds to more fluctuation as a function of $L$ than the 0.5 case, and therefore to a more tortuous channel. 

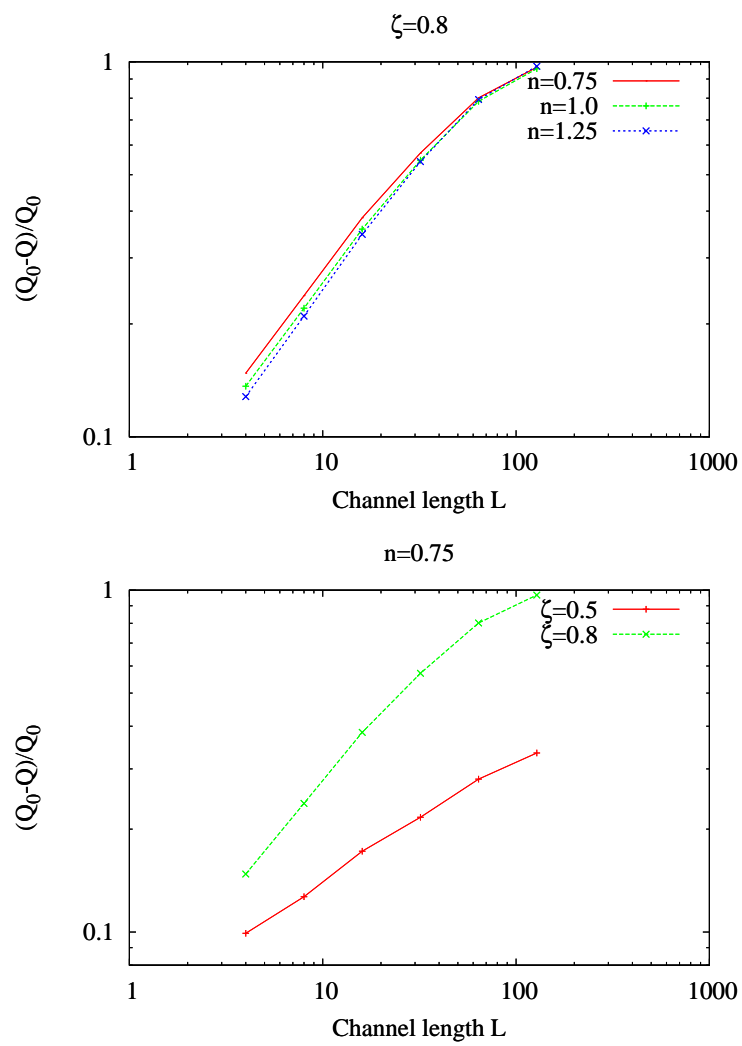

FIG. 12: Flux variation with length in a channel with one self-affine and one flat wall for different fluids and Hurst exponents. The maximum aperture of the channel is $H_{\max }=64$, and the applied pressure gradient is $G=1.0 \times 10^{-6}$. Top: flux depletion for different fluids confined in a channel with $\zeta=0.8$. Right: flux depletion for a shear-thinning fluid in channels of different $\zeta$.

To relate the flux to the channel aperture, we imagine dividing the channel into a sequence of nearly-straight sections, each of length $\ell_{i}$, and writing the total pressure difference as the sum of the pressure drops in each section, using Eq. 8 for each. This reasoning yields

$\Delta P=\sum_{i} \Delta P_{i}=\sum_{i}\left[Q \cdot \frac{2 n+1}{n} \cdot b_{i}^{-\frac{2 n+1}{n}}\right]^{n} \cdot m \cdot 2^{n+1} \cdot l_{i}$,

where the summation is over the sections, and $b_{i}$ is the effective aperture and $l_{i}$ is the length along the local flow direction in section $i$, and we have noted that $Q$ is a the same in all sections. If $\theta_{i}$ is the angle between the orientation of channel section $i$ and the mean flow direction, then $b_{i}=H \cos \theta_{i}$ and $l_{i}=l_{i}^{\|} / \cos \theta_{i}$, where $H$ is the aperture and $l^{\|}$is the projected length of section $i$ in the mean flow direction, assumed to be the same for all sections. Using these relations in Eq15 we have

$$
\Delta P=2 m Q l^{\|}\left[2 \frac{2 n+1}{n} H^{-\frac{2 n+1}{n}}\right]^{n} \sum_{i}\left(\cos \theta_{i}\right)^{-(2 n+2)} .
$$

This result generalizes Eq. 26 in [6] to power-law fluids, and if we proceed as in that reference to evaluate the average over angles $\theta_{i}$ we obtain

$$
Q-Q_{0} \sim H^{(2 \zeta-2) / \zeta+(2 n+1) / n}
$$

where again $Q_{0}$ is the flux in a flat channel of the same aperture $H$.

To test the relation 17, we calculate the flow for fracture channels of length $L=256$ with varying apertures $H=8,12,16,20,24$, for fluid with $m=0.01, n=0.75,1.0$ and 1.25 , all at a pressure gradient $\Delta P / L=1.0 e-6$. Figure 13 shows the flux depeltion $\left(Q_{0}-Q\right)$ as a function of aperture. The points are the numerical results and the solid lines are fitted curves, based on the expected power-law exponents obtained from Eq17, which are $2.83,2.5$, and 2.3 for the three fluids. We see that the theoretical analysis is in excellent agreement with the data for the shear-thinning and Newtonian fluids ( $n=0.75$ and 1.0), but the agreement is less statisfactory for the shear-thickening fluid, whose numerical exponent is closer to 2.5. A possible interpretation is that in the shear-thickening case, for the same pressure gradient the average velocity is larger than that for the other fluids, so that fluid inertia comes into play.

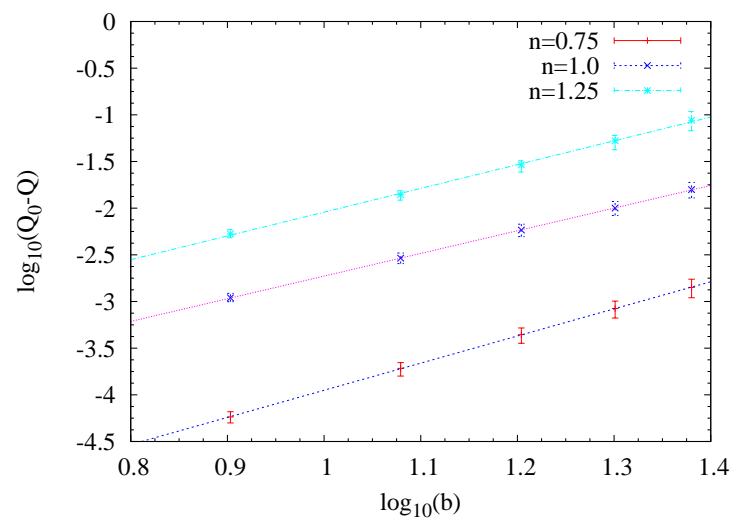

FIG. 13: Log-log plot of flow rate variations versus the aperture of self-affine channel for different fluids with power $n=0.75,1.0$ and 1.25 .

Finally, we consider an additional effect, a lateral shift between the two sides of a fracture, which might arise in practice due to geological processes. We begin with a fracture channel with complimentary sides and constant initial aperture $H$, and then shift one side along the mean plane by a distance $d$. The fracture aperture is now a function of position, $H_{d}(x)$, and effectively a spatial random function. We again compute the flux depletion relative to a flat channel having the same ini- 
tial aperture, using six realizations of a self-affine fracture wall with Hurst exponent $\zeta=0.8$. As shown in Fig. 14 the flux decreases somewhat faster than linearly with shift, by producing narrow gaps when proturbances on the two sides are brought closer to one another. The shear-thinning and Newtonian fluids have a fairly similar behavior, while the reduction is twice as large in the shear-thickening case, perhaps again as a result of inertial effects. As in the previous discussion, using a different value $\zeta=0.5$ for the Hurst exponent gives the same trends.

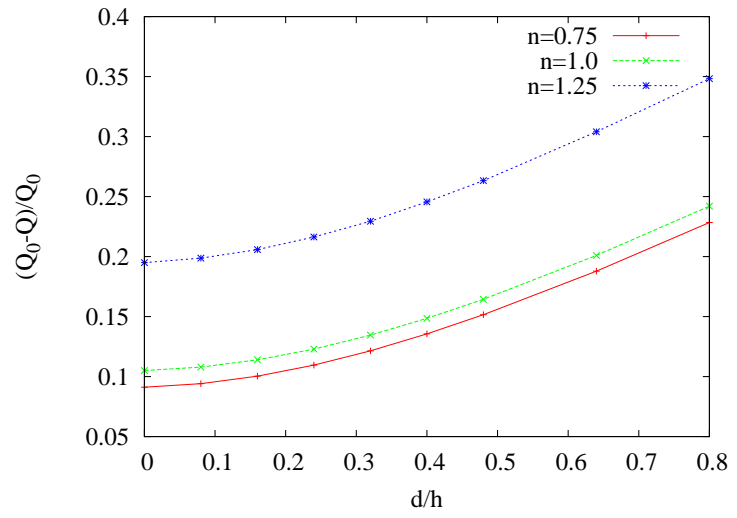

FIG. 14: Flow rate reduction due to lateral shift along the mean plane of fracture channel for different fluids with power $n=0.75,1.0$ and 1.25, and the Hurst exponent used here is $\zeta=0.8$.

\section{CONCLUSION}

Using a new implementation of the lattice Boltzmann method for power-law fluids, we have investigated their flow in two-dimensional self-affine fracture channels as a function of applied pressure gradient. Generally, fluids with different power-law index behave in a similar manner when their flow parameters are properly scaled, using standard results for flow in constant-thickness channels. Many previous results for Newtonian fluids in selfaffince fractures are found to generalize in a straightforward manner. However, shear-thickening fluids, which have higher velocities for the same pressure gradient than Newtonian or shear-thnning counterparts, are more susceptible to inertial effects.

With regard to the local flow fields, we first considered the maximum absolute velocity as a function of dis- tance along the mean flow direction, which was found to fluctuates along the fracture channel due to its tortuosity and the variable effective aperture along the channel. The local maxima of this maximum absolute velocity occur at points of narrowing or minimal effective aperture, and the range of maximum absolute velocity relative to the global mean velocity ranges from about 1.5 to 5.5 . With increasing inertia, this normalized maximum absolute velocity increases for all power-law fluids to different degrees, with shear-thickening fluids having the largest effect and shear-thinning the least. As the pressure gradient increases, the normalized maximum velocities near the constrictions are relatively constant but outside these points velocities tend to increase. The variation in velocity is greatest for a shear-thickening fluid and lest for shear-thinning. Pressure fluctuations along the channel increase with forcing for all fluids, and for a given pressure gradient increase with the power-law index $n$.

The relationship between pressure gradient and flux is found to have the same functional form as for flow in a flat channel, $\Delta p \sim Q^{n}$, when inertial effects are absent. At higher $\Delta p$, Newtonian fluids behave in the same way as in intergranular porous media, and shear-thinning fluids behave analogously, but the shear-thickening case does not show simple power-law behavior. It is possible to collapse all of the data on flux vs. pressure gradient into a universal friction factor curve. The variation of flux with system length was shown to scale with system length with an exponent algebraically related to the Hurst exponent, in a manner which generalizes the Newtonian case.

The most interesting question raised by these results is the form of the flux-pressure gradient relationship in the regime of strong inertia in the non-Newtonian case. In this work, we were limited in the range of accessible pressure gradients by numerical instabiliites, and it is desirable to improve the algorithm so as to consider higher pressure gradients and further explore the dynamics of the inertial regime. An extension of these considerations to viscoelastic fluids is likewise highly desirable, but new ideas beyond the methods used here are needed.

\section{Acknowledgments}

This work was supported by the NSF CREST Center for Mesoscopic Modeling and Simulation at CCNY, and the Geosciences Program of the Office of Basic Energy Sciences, U. S. Department of Energy. We thank German Drazer and Jean-Pierre Hulin for helpful discussions and suggestions.
[1] P. M. Adler and J.-F. Thovert, Fractures and Fracture Networks (Kluwer, Dordrecht, 1999).

[2] B. Berkowitz, Adv. Water Resources 25, 861 (2002).

[3] P. Dietrich et al., eds., Flow and Transport in Fractured
Porous Media (Springer, Berlin, 2005).

[4] M. Sahimi, Flow and Transport in Porous Media and Fractured Rocks (Editions VCH, Weinheim, 1995).

[5] E. Bouchaud, J. Phys.: Condens. Matter 9, 4319 (1997). 
[6] G. Drazer and J. Koplik, Phys. Rev. E 62, 8076 (2000).

[7] G. Drazer and J. Koplik. Phys. Rev. E 66, 026303 (2002).

[8] A. Hansen E. Skjetne and J.S. Gudmundsson, J. Fluid Mech.383, 1 (1999)

[9] A. Boschan, H. Auradou, I. Ippolito, R. Chertoff and J. P. Hulin, Water Resources Res. 43, W03438 (2007).

[10] I. Machac R. P. Chhabra, J. Comiti, Chem. Engr. Sci. 56, 27 (2001).

[11] G. Drazer S. Gabbanelli and J. Koplik, Phys. Rev. E 72, 046312 (2005).

[12] J. Feder, Fractals (Plenum, New York, 1988).

[13] R. F. Voss, in Fundamental Algorithms in Computer graphics (Springer-Verlag, Berlin, 1985), pp. 805-835.

[14] S. Succi, The Lattice Boltzmann Equation for Fluid Dynamics and Beyond (Clarendon Press, Oxford, 2001).

[15] R. Verberg and T. Ladd, J. Stat. Phys. 104, 1191 (2001).
[16] D. H. Rothman and E. Aharonov, Geophys. Res. Lett. 20, 679 (1993).

[17] S. Chen and G. D. Doolen, Annu. Rev. Fluid Mech. 30, 329 (1998).

[18] M. L. Johns S. P. Sullivan, L. F. Gladden, J. NonNewtonian Fluid Mech. 133, 91 (2006).

[19] E. Guyon, J.-P. Nadal and Y. Pomeau, Disorder and Mixing (Kluwer, Dordrecht, 1988).

[20] J. Bear, Dynamics of Fluids in Porous Media (New York, Elsevier, 1972).

[21] D. L. Koch and R. J. Hill, Annu. Rev. Fluid Mech. 33, 619 (2001).

[22] H. Schlichting and K. Gersten, Boundary Layer Theory, 8th ed. (Springer, Berlin, 2000).

[23] A. B. Metzner, Adv. Chem. Eng. 1, 77 (1956) 